\title{
Altered matrix production in the distal airways of individuals with asthma
}

\author{
Kristian Nihlberg, ${ }^{1}$ Annika Andersson-Sjöland, ${ }^{2}$ Ellen Tufvesson, ${ }^{2}$ Jonas S Erjefält, ${ }^{1}$ \\ Leif Bjermer, ${ }^{2}$ Gunilla Westergren-Thorsson ${ }^{1}$
}

- Supplementary methods and a figure are published online only. To view these files please visit the journal online (http:// thorax.bmi.com).

${ }^{1}$ Department of Experimental Medical Science, Division of Vascular and Airway Research, LungBiology, Lund University, Sweden

${ }^{2}$ Department of Clinical Medical Sciences, Lund, Division of Respiratory Medicine and Allergology, Lund University, Sweden

\section{Correspondence to}

Kristian Nihlberg, Experimental Medical Science, BMC D12.

Klinikgatan 32, Lund 221 84, Sweden;

kristian.nihlberg@med.lu.se

Received 21 October 2009 Accepted 8 May 2010

\section{ABSTRACT}

Background and aims Although increasing evidence suggests involvement of the distal airway in all stages of asthma, it is not known whether structural changes (defined as airway remodelling) occur in the distal airways of subjects with mild asthma and those with atopy. The aim of this study was to compare control subjects and those with mild asthma in relation to fibroblast phenotypes and remodelling in central and distal airways.

Methods Distal and central fibroblasts from controls $(n=12)$ and patients with mild asthma $(n=11)$ were cultured and incubated for $24 \mathrm{~h}$ with $0.4 \%$ serum, or stimulated with transforming growth factor $\beta 1$ (TGF $\beta 1$ ). $\left[{ }^{35} \mathrm{~S}\right]$ Sulfate-labelled proteoglycans in culture medium were analysed by ion exchange chromatography and sodium dodecyl sulfate-polyacrylamide gel electrophoresis. Proliferation was measured with crystal violet, and exhaled nitric oxide was measured by the fractional nitric oxide technique.

Results Vesican production from distal fibroblasts was significantly elevated in patients with asthma compared with controls ( $p<0.001)$, and the percentage collagenpositive area in distal asthma tissue was also enhanced compared with controls $(p<0.01)$. In addition, distal asthma fibroblasts had reduced proliferation capacity compared with those of controls (by $24 \% ; p<0.01$ ). Furthermore, the alveolar nitric oxide concentration was correlated to distal biglycan and perlecan production of subjects with asthma $(r=-0.857, p<0.05$ and $r=-0.750, p<0.05$ respectively)

Conclusion It is shown that centrally and distally derived fibroblasts differ in their proteoglycan production and proliferation between central and distal tissue, and in those with asthma compared with controls. It is also demonstrated that remodelling is present in distal lung of subjects with mild asthma. This may be of importance in airway remodelling and asthma progression.

\section{INTRODUCTION}

In the pathogenesis of asthma, the alterations in the central airways are well characterised and involve inflammatory and connective tissue changes with proposed implications for decline in lung function and increased bronchial hyperresponsiveness. However, there are some recently published data to suggest that the distal airways (ie, $<2 \mathrm{~mm}$ conducting airways and lung parenchyma) may play a role in the pathophysiology of asthma. ${ }^{1-3}$ Structural pathological changes are collectively called remodelling. In asthma, typical features include thickening of the reticular epithe- lial basement membrane, subepithelial fibrosis, epithelial shedding and smooth muscle hypertrophy/hyperplasia. ${ }^{4}$ In this process, the extracellular matrix-producing fibroblast-myofibroblast phenotypes deposit molecules such as collagen and proteoglycans. $^{5-7}$ Proteoglycans are a family of sulfated molecules consisting of a core protein that is associated with one or several glycosaminoglycan chains. The matrix-associated proteoglycans are mainly the connective tissue-associated versican, the large basement membrane proteoglycan perlecan and the small proteoglycans biglycan and decorin. ${ }^{8}$ These groups of molecules are of major importance in tissue elasticity and directly affect fluid balance and collagen assembly, and also the activities and storage of cytokines and growth factors such as transforming growth factor $\beta 1$ (TGF 11$)^{9}{ }^{10}$ Activation of fibroblasts leads to a shift in fibroblast phenotype. This has been studied in other fibrotic disorders such as kidney fibrosis, systemic sclerosis and idiopathic pulmonary fibrosis. ${ }^{11} 12$ Interestingly, fibroblasts from different airway levels seem to differ in their phenotype. This was shown, for example, in a study in which cultured nasal and bronchial fibroblasts responded differently to TGF $\beta 1$, and their matrix production was compared. ${ }^{13}$ In addition to changes in matrix turnover, upregulation of exhaled nitric oxide (NO) is also increased in asthma, systemic sclerosis and allergic alveolitis, and this parameter is suggested to be a reflection of the increased inflammation. ${ }^{14}$ Consequently, fractional exhaled nitric oxide (FeNO) is frequently used in the clinic and is believed to be associated with eosinophilic inflammation in the central airways. ${ }^{15} 16$ The airway epithelium and leucocytes such as macrophages are sources of exhaled $\mathrm{NO}$, but it is likely that other cells such as fibroblasts may also be of importance in increased $\mathrm{NO}$ production.

To explore whether fibroblasts from different airway levels in the human lung have adopted distinct disease-related and location-dependent phenotypes, we cultured primary central and distal fibroblasts from subjects with mild asthma and controls without asthma. The control subjects were divided further into two groups based on whether or not they were atopic. Atopic features may precede the onset of asthma, and this is therefore a patient group of interest. In addition, treatment of allergic rhinitis in subjects with asthma may improve co-existing asthma. ${ }^{17}$

We hypothesised that distal lung fibroblasts are different from centrally derived fibroblasts regarding proteoglycan production, proliferation 
and morphology. We also wanted to investigate whether differences in fibroblast phenotypes could be observed between those without asthma and those with mild asthma, and whether they could be correlated to inflammatory parameters such as NO.

\section{MATERIALS AND METHODS Subjects and sampling of tissue}

Twenty-nine individuals were enrolled in the study (13 patients with asthma and 16 controls). In six individuals, fibroblast culture was not established and they were therefore excluded from further analysis. Eleven fibroblast cultures were established from patients with asthma with definitive bronchial hyperresponsiveness, defined as $\mathrm{PD}_{20}$ (provocative dose causing a $20 \%$ fall in forced expiratory volume in $\left.1 \mathrm{~s}\left(\mathrm{FEV}_{1}\right)\right)<2000 \mu \mathrm{g}$ methacholine, and they all had mild asthma according to Global Initiative for Asthma (GINA) guidelines. ${ }^{18}$ All individuals were free from infections and had not used any corticosteroid treatment from 3 months prior to the start of the study. Healthy agematched non-smoking subjects were used as controls. They all denied having respiratory symptoms, and were all methacholine challenge test negative. Seven of the controls were non-atopic and five were atopic, as defined by confirmed sensitisation by skin prick test to one or more allergens (birch, timothy, mug wort, cat, dog, and/or house dust mite). ${ }^{19}$ The atopic subjects also had rhinitis. Central bronchoscopy was performed with a flexible bronchoscope (Olympus IT60, Tokyo, Japan) followed by a transbronchial biopsy specimen taken with biopsy forceps (Olympus FB211D) guided by a fluoroscope. As local anaesthesia, lidocaine spray was given through a spray catheter. All subjects gave written informed consent and the study was approved by the ethics committee of Lund University (LU412$03)$. For more information see the supplementary data online.

\section{Cell cultures and morphological characterisation}

For morphological characterisation, crystal violet staining was used as previously described. ${ }^{10}$ For further characterisation of fibroblasts, cells were labelled with antibodies to vimentin, prolyl-4-hydroxylase or SM22, and incubated with appropriate fluorescent-labelled secondary antibodies. For counting of cellular extensions, 250 cells/subject were randomly counted. For more information see the supplementary data online.

\section{Trichrome staining}

Sections of periodate-paraformaldehyde-lysine (PLP)-fixed transbronchial biopsies were stained with Masson's trichrome stain for measurement of collagen content in the alveolar parenchyma. Blue collagen staining was analysed by computerised image analysis (ImageJ, version 1.41, NIH). For a detailed description, see the supplementary data online.

\section{Proliferation assay}

Cells were seeded in 96-well plates. Non-adherent cells were washed out after $6 \mathrm{~h}$ of incubation and fresh medium was added for the remaining $24 \mathrm{~h}$. Cells were fixed, and stained with crystal violet. For more information see the supplementary data online.

\section{Proteoglycan analysis}

Cells were grown in ${ }^{35} \mathrm{~S}$-supplemented medium, and diethylaminoethyl (DEAE)-52 cellulose anion exchange resin columns were used to extract the proteoglycans. The individual proteoglycans were separated further on a sodium dodecyl sulfate (SDS)-polyacrylamide gel and quantification of the amount of proteoglycan was done using a scintillation counter as previously described; see the supplementary data online.

\section{NO measurement}

Measurements were performed as previously described. ${ }^{18}$ Briefly, FeNO measurements were done prior to bronchial challenge testing at a flow rate of 50,100, 200 and $400 \mathrm{ml} / \mathrm{s}$ using a NIOX nitric oxide analyser (Aerocrine AB, Stockholm, Sweden), and the results were expressed as parts per billion (ppb). Alveolar NO concentration and bronchial flux of $\mathrm{NO}$ were calculated with a two-compartment linear model using a flow rate of $100-400 \mathrm{ml} / \mathrm{s}$.

\section{Statistical methods}

For non-parametric data, between-groups comparisons were performed with the Kruskal-Wallis test (a non-parametric test on ranks, which compares the means of three or more unmatched groups) with the Dunn multiple comparison test followed by the Mann-Whitney $U$ test. Correlations were tested by Spearman rank correlation using Graph pad software version 5.03 (San Diego, California, USA). For parametric data the Student $t$ test was used. If not all subjects are represented in the graphs, these have been excluded due to technical problems such as loss of biopsies when staining tissue or limited amount of material. Data are presented as median values together with the range unless otherwise stated; $p$ values of $<0.05\left(^{*}\right)$ and $<0.01\left({ }^{* *}\right)$ denote significant levels of difference.

\section{RESULTS}

\section{Patient characterisation and fibroblast recovery}

Of the 29 individuals originally considered for the study, fibroblast cultures were established from 23 (11 patients with asthma and 12 controls). Subjects with asthma were 22-38 years of age (mean 28) and controls were 22-40 (mean 28). No differences in $\mathrm{FEV}_{1}$ or $\mathrm{FEV}_{1} \%$ predicted were seen when comparing those with asthma and controls (4.2 vs 3.9 litres and $95 \%$ vs $104 \%$, respectively). However, the mean FeNO50 (exhaled $\mathrm{NO}$ at $50 \mathrm{ml} / \mathrm{s}$ ) of those with asthma was significantly elevated relative to that of the controls (75.8 (15.8-240) ppb vs 15.4 (8.4-27.7) $\mathrm{ppb}), \mathrm{p}=0.017)$ and relative to that of the atopic controls without asthma (75.8 (15.8-240)) ppb vs 16.6 (9.8-27.7) ppb), $\mathrm{p}=0.018$ ). For bronchial flux of NO, subjects with asthma had levels that were sixfold higher than those of the controls (4.61 $(0.68-12.4)$ vs $0.73(0.38-1.28), \mathrm{p}=0.007)$ and also higher compared with atopic controls $(4.61(0.68-12.4)$ vs $(0.81$ (0.47-1.28), $\mathrm{p}=0.024$ (table 1).

\section{Increased remodelling in tissue from distal airways in patients with mild asthma}

Distal lung biopsies were stained by trichrome staining to visualise collagen regions in the alveolar parenchyma (exemplified in figure 1A, B, black arrows). Elongated spindle-shaped collagen areas were clearly visualised in distal biopsies especially from patients with asthma (figure 1B). Distal lung collagen expression was significantly elevated in patients with asthma compared with controls $(6.9(3.6-8.1)$ vs 3.8 (1.42-6.2), $\mathrm{p}=0.007)$. No difference was seen between atopic and nonatopic controls (figure 1C).

\section{Characterisation of fibroblasts}

Distal fibroblasts were all positive for the filament proteins vimentin (figure $2 \mathrm{~A}, \mathrm{E}$ ) and $\boldsymbol{\alpha}$-smooth muscle actin (figure $2 \mathrm{~B}, \mathrm{~F}$ ). Furthermore, these cells also expressed prolyl-4-hydroxylase, 
Table 1 Clinical characterisation of subjects included in the study and fibroblasts successfully cultured from different locations of the lung from these subjects

\begin{tabular}{|c|c|c|c|c|c|c|c|c|c|c|c|}
\hline Subjects & $\begin{array}{l}\text { Asthma/ } \\
\text { control }\end{array}$ & $\begin{array}{l}\text { Gender } \\
\text { (M/F) }\end{array}$ & $\begin{array}{l}\text { Age } \\
\text { (years) }\end{array}$ & $\begin{array}{l}P_{20} \\
(\mu g)\end{array}$ & $\begin{array}{l}\text { Atopy } \\
\text { (Y/N) }\end{array}$ & $\begin{array}{l}\mathrm{FEV}_{1} \\
\text { (litres) }\end{array}$ & $\begin{array}{l}\mathrm{FEV}_{1} \% \\
\text { predicted }\end{array}$ & $\begin{array}{l}\text { FeN050 } \\
\text { (ppb) }\end{array}$ & $\begin{array}{l}\text { Bronchial } \\
\text { flux }(\mathrm{nl} / \mathrm{s})\end{array}$ & $\begin{array}{l}\text { Alveolar } \\
\text { NO (ppb) }\end{array}$ & $\begin{array}{l}\text { Fibroblasts } \\
\text { (C/D) }\end{array}$ \\
\hline 1 & Asthma & M & 38 & 122 & $Y$ & 4.43 & 98 & 239.7 & 12.39 & 5.62 & $C / D$ \\
\hline 2 & Asthma & $M$ & 22 & 24 & $Y$ & 6.04 & 124 & 100.6 & 4.91 & 4.37 & $C / D$ \\
\hline 3 & Asthma & $\mathrm{F}$ & 23 & 5 & $Y$ & 3.41 & 92.4 & 109.3 & 5.69 & 3.60 & $C / D$ \\
\hline 4 & Asthma & $\mathrm{F}$ & 31 & 16 & $Y$ & 3.06 & 94.8 & 61.7 & 0.91 & 2.85 & $C / D$ \\
\hline 5 & Asthma & M & 24 & 78 & $Y$ & 7.31 & 91.8 & 18.9 & 3.55 & 3.64 & $D$ \\
\hline 6 & Asthma & M & 24 & 79 & $Y$ & 3.81 & 88 & 69.4 & 4.19 & 2.09 & $D$ \\
\hline 7 & Asthma & $\mathrm{F}$ & 28 & 145 & $Y$ & 3.16 & 103 & 15.8 & 0.68 & 2.96 & $D$ \\
\hline 8 & Asthma & M & 25 & 134,5 & $Y$ & 4.51 & 90 & ND & ND & ND & C \\
\hline 9 & Asthma & M & 35 & $<0.05$ & $Y$ & 2.21 & 62 & 32,2 & ND & ND & C \\
\hline 10 & Asthma & $F$ & 31 & $<0.05$ & $Y$ & 3.36 & 102 & 44 & ND & ND & C \\
\hline 11 & Asthma & $\mathrm{F}$ & 26 & 826 & $Y$ & 4.57 & 101 & ND & ND & ND & C \\
\hline 12 & Control & M & 28 & $>2000$ & $Y$ & 3.98 & 108.7 & 21.20 & 1.16 & 2.34 & $C / D$ \\
\hline 13 & Control & $\mathrm{F}$ & 27 & $>2000$ & $Y$ & 2.98 & 96.3 & 9.80 & 0.47 & 1.74 & $C / D$ \\
\hline 14 & Control & $\mathrm{F}$ & 37 & $>2000$ & $Y$ & 3.21 & 103.8 & 15.60 & 0.56 & 3.74 & $D$ \\
\hline 15 & Control & $\mathrm{F}$ & 24 & $>2000$ & $Y$ & 3.21 & 105 & 13.2 & 0.6 & 2.66 & $C / D$ \\
\hline 16 & Control & $M$ & 29 & $>2000$ & $Y$ & 4.69 & 114.6 & 27.7 & 1.28 & 3.0 & $C / D$ \\
\hline 17 & Control & M & 30 & $>2000$ & $\mathrm{~N}$ & 5.11 & 116.4 & 17.3 & 0.89 & 2.27 & $D$ \\
\hline 18 & Control & $\mathrm{F}$ & 24 & $>2000$ & $\mathrm{~N}$ & 3.68 & 95.4 & 9.8 & 0.44 & 2.48 & $D$ \\
\hline 19 & Control & M & 40 & $>2000$ & $\mathrm{~N}$ & 4.91 & 109.8 & 8.4 & 0.38 & 2.13 & $C / D$ \\
\hline 20 & Control & M & 24 & $>2000$ & $\mathrm{~N}$ & 5.38 & 113.5 & 21.10 & 1.10 & 3.70 & $C / D$ \\
\hline 21 & Control & $F$ & 33 & $>2000$ & $\mathrm{~N}$ & 3.71 & 100.7 & 16.10 & 1.02 & 2.02 & $C / D$ \\
\hline 22 & Control & $\mathrm{F}$ & 22 & $>2000$ & $\mathrm{~N}$ & 2.38 & 95.1 & 8.90 & 0.70 & 1.68 & $\mathrm{D}$ \\
\hline 23 & Control & $\mathrm{F}$ & 23 & $>2000$ & $\mathrm{~N}$ & 3.25 & 84.1 & 16.60 & 0.75 & 2.10 & $D$ \\
\hline
\end{tabular}

C, successful recovery of central fibroblasts; $\mathrm{D}$, successful recovery of distal fibroblasts; $\mathrm{F}$, female; FeN050, exhaled NO at $50 \mathrm{ml} / \mathrm{s}$; FEV 1 , forced expiratory volume in $1 \mathrm{~s}$; $\mathrm{M}$, male; $\mathrm{N}$, no; $\mathrm{ND}$, not done; $\mathrm{NO}$, nitric oxide; $\mathrm{PD}_{20}$, metacholine responsiveness $(2000 \mu \mathrm{g}$ represents the threshold); $\mathrm{Y}$, yes.

which is a key enzyme required for the post-translational hydroxylation of proline residues in collagen (figure $2 \mathrm{C}, \mathrm{G}$ ). The cells were negative for one of the most sensitive markers of smooth muscle cells, SM22, further supporting the idea that we had cultured a homogenous fibroblast population (figure $2 \mathrm{D}, \mathrm{H}$ ). The same pattern of staining was seen for the centrally derived fibroblasts (data not shown), which has also been shown previously. $^{35}$
Figure 1 Collagen in distal airways in patients with mild asthma.

Representative images of collagen in distally derived biopsies from a control subject $(A)$ and a patient with asthma (B) visualised with Masson trichrome staining $\times 400$ magnification. Black arrows indicate collagen (blue) in the parenchyma from a control and a patient with asthma. Quantitative data of tissue collagen content in controls and subjects with asthma are shown in (C). Mann-Whitney tests were used to calculate $\mathrm{p}$ values. Values are

expressed as collagen-positive area as a percentage of the total tissue area.

Because of loss of biopsies (detached from the slide) in the staining procedure collagen was not analysed in two of the atopic subjects and in two of the nonatopic controls. Open circles represent non-atopic controls $(n=5)$, grey circles represent atopic controls $(n=3)$ and filled circles represent subjects with asthma $(n=7)$. Mann-Whitney tests were used to calculate significant differences between controls and patients with asthma.
A

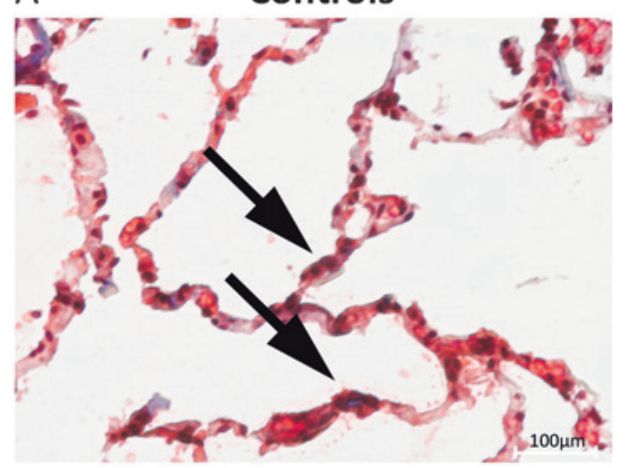

C

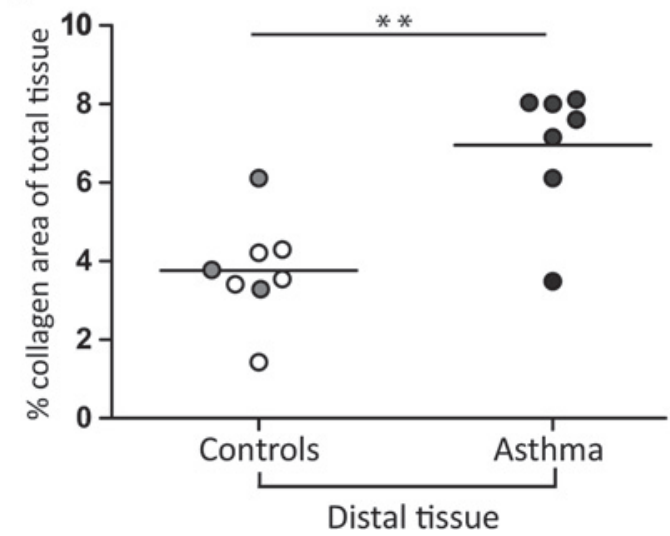

B Asthma

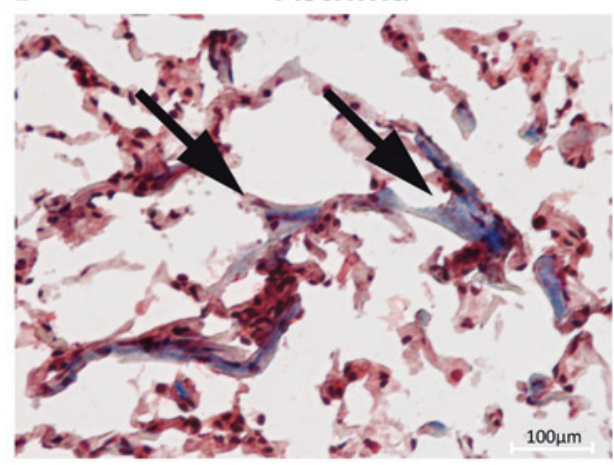



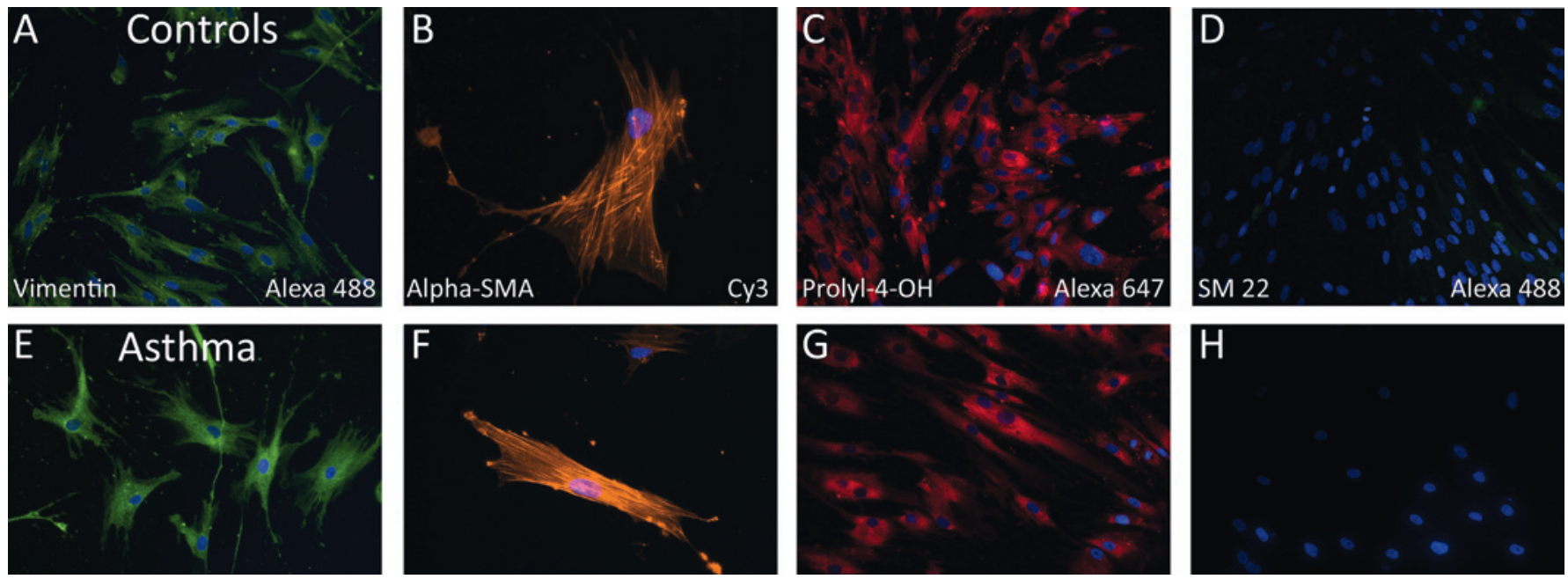

Figure 2 Characterisation of distal fibroblasts. Distal fibroblasts were cultured for $48 \mathrm{~h}$ and stained for mesenchymal and smooth muscle proteins to investigate fibroblasts from controls and patients with asthma. Cells from control individuals $(A-D)$ and patients with asthma (E-H) were all positive for the mesenchymal fibroblast protein vimentin (green, Alexa 488), $\alpha$-smooth muscle actin (yellow, Cy3) and prolyl-4-hydroxylase (red, Alexa 647) but negative for the smooth muscle marker SM22 (green, Alexa 488). Staining of nuclei is visualised in blue (4',6-diamidino-2-phenylindole (DAPI)).

Distally and centrally derived fibroblasts from subjects with asthma have reduced proliferation capacity

Centrally derived fibroblasts from subjects with asthma had $24 \%$ lower proliferation capacity relative to the controls, after $24 \mathrm{~h}(1.06(0.96-1.15)$ vs $1.39(1.08-1.88), \mathrm{p}=0.004)$ (figure 3A). This was also seen with distally derived asthma fibroblasts, which had a lower proliferation rate (23\%) compared with the corresponding controls (1.27 (1.12-1.57) vs 1.65 (1.23-2.06), $\mathrm{p}=0.002$ ) (figure $3 \mathrm{~A}$ ). There was a significant increase in fibroblast proliferation when comparing central versus distal in both controls and subjects with asthma (1.39 (1.08-1.88) vs 1.65 $(1.23-2.06), p=0.034$, and $1.06(0.96-1.15)$ vs $1.27(1.12-1.57)$, $p=0.004$, respectively) (figure $3 \mathrm{~A}$ ). The same difference in proliferation could also be measured after $48 \mathrm{~h}$ (data not shown).
Central fibroblasts from subjects with asthma have more cellular extensions than distal fibroblasts

When counting the number of cellular extensions in centrally derived fibroblasts, cells from subjects with asthma had significantly more (1.4 times more) cellular extensions than those from controls $(8.3(4-20)$ vs $5.9(2-20), \mathrm{p}<0.001)$, figure $3 \mathrm{~B}$, black arrows). Distally derived fibroblasts were more elongated and had fewer extensions than centrally derived fibroblasts (2.6 $(1-7)$ vs $6.6(2-20), \mathrm{p}<0.001)$.

Increased matrix production by distally and centrally derived fibroblasts is associated with disease

Production of the large molecule versican was significantly greater in centrally derived fibroblasts from those with asthma than in control fibroblasts (354 (201-571) vs $96(18-201) \mathrm{dpm}$
A

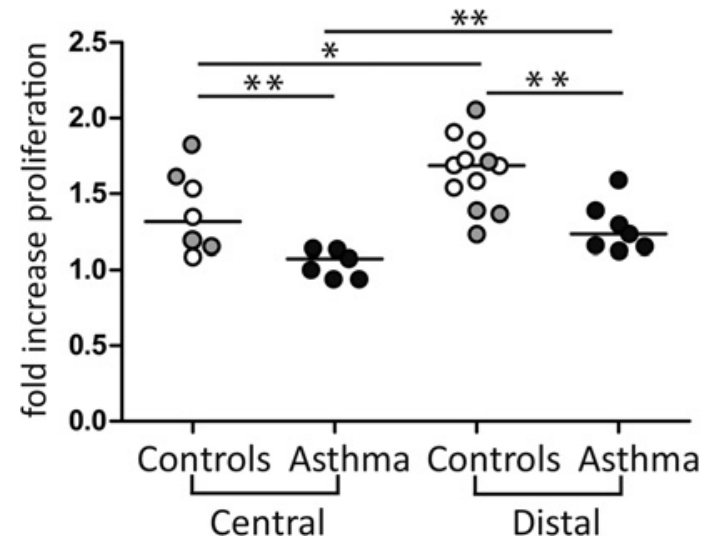

B

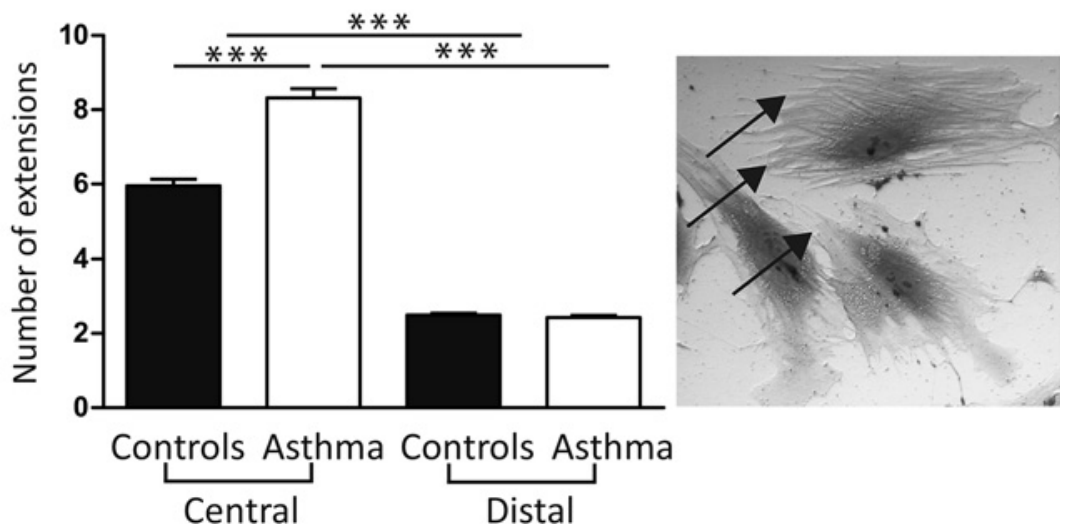

Figure 3 Culture and morphological characterisation of centrally and distally derived fibroblasts. (A) Fibroblasts from central and distal locations were seeded in 96 -well plates at a density of 5000 cells/well and cultured for $24 \mathrm{~h}$. Glutaraldehyde-fixed cells stained with crystal violet were lysed with $1 \%$ Triton X-100 and the absorbance measured at $595 \mathrm{~nm}$. (B) Each cell was counted for cellular extensions (black arrows). In two of the patients with asthma, proliferation of centrally derived fibroblasts could not be measured because of a limited amount of material. Student $t$ test was used to calculate $\mathrm{p}$ values. Open circles represent non-atopic controls, grey circles represent atopic controls and filled circles represent subjects witha sthma. ${ }^{*}$, Significant difference $(\mathrm{p}<0.05)$ when comparing proliferation of centrally derived versus distally derived fibroblasts from controls. ${ }^{* *}$, Significant difference $(p<0.01)$ when comparing distally derived fibroblasts from controls versus patients with asthma, when comparing centrally control fibroblast versus centrally asthma fibroblasts, and when comparing central asthma versus distal asthma fibroblasts. 
Figure 4 Proteoglycan production in centrally and distally derived fibroblasts. Centrally and distally derived fibroblasts from controls and from subjects with asthma were analysed for proteoglycan production: (A) versican; (B) biglycan; (C) perlecan; and (D) decorin. Cells were grown in ${ }^{35} \mathrm{~S}$-supplemented sulfate-poor medium, and diethyl-aminoethyl (DEAE)-52 columns were used to extract the proteoglycans. Quantification of proteoglycan production was done using a scintillation counter and is presented as $\mathrm{dpm} / \mu \mathrm{g}$ protein. The individual proteoglycans were separated further by sodium dodecyl sulfate-polyacrylamide gel electrophoresis (SDS-PAGE).

Mann-Whitney tests were used to calculate $p$ values. Values presented are medians, and open circles represent non-atopic controls (central $n=3$, distal $n=7)$, grey circles represent atopic controls (central $n=4$, distal $n=5$ ) and filled circles represent subjects with asthma (central $n=8$, distal $n=7$ ). ** Statistically significant difference $(p<0.01)$ for versican $(A)$ and biglycan (B) production by centrally derived fibroblasts when comparing controls and patients with asthma. ${ }^{* *}$. Significant difference $(\mathrm{p}<0.001)$ for distal versican production (controls vs asthma patients). ${ }^{*}$ and \#, Significant difference $(p<0.05)$ for central biglycan production versus distal production from controls and for distal biglycan and decorin production (non-atopic controls vs patients with asthma (open circle vs filled circle) and non-atopic controls versus atopic controls (open circle vs grey circle)).

(disintegrations $/ \mathrm{min}) / \mu \mathrm{g}, \mathrm{p}<0.01$, figure $4 \mathrm{~A}$ ). Consistent with data from the central airways, distally derived fibroblasts from subjects with asthma also had considerably higher versican production than control fibroblasts $(210(163-259)$ vs 106 $(45-162) \mathrm{dpm} / \mu \mathrm{g}, \mathrm{p}<0.001$, figure $4 \mathrm{~A})$. In control subjects, there was no difference between atopic and non-atopic subjects (figure $4 \mathrm{~A}$ ). Expression of the small leucine-rich proteoglycan biglycan was studied further. We found that there was enhanced biglycan production from centrally derived fibroblasts from those with asthma compared with controls (163 (84-346) vs 81 (47-144) $\mathrm{dpm} / \mu \mathrm{g}, \mathrm{p}=0.006$, figure 4B). These differences could not be detected in distal airway fibroblasts. However, biglycan production by distally derived fibroblasts from patients with asthma was significantly lower than from non-atopic controls (145 (72-207) vs $349(224-480) \mathrm{dpm} / \mu \mathrm{g}, \mathrm{p}<0.001$, figure 4B). Interestingly, atopic controls also had significantly lower production of biglycans than non-atopic controls (122 (40-255) vs $349(224-480) \mathrm{dpm} / \mu \mathrm{g}, \mathrm{p}=0.010)$ (figure $4 \mathrm{~B}$ ). The large $(>400 \mathrm{kDa}$ ) multidomain proteoglycan perlecan which is mainly localised in the basement membrane was not different in expression regardless of whether central or distal localisation or different groups were compared (figure 4C). As observed for distal biglycan production, decorin production in both patients with asthma and atopic controls was significantly lower than in non-atopic controls (127 (56-258) and 109 (66-154) vs 343 (55-476) dpm/ $\mu \mathrm{g} ; \quad \mathrm{p}=0.026$ and $\mathrm{p}=0.048$, respectively) (figure 4D).

\section{Alveolar NO was correlated to production of individual proteoglycans in distal airways}

Negative correlations were observed when comparing alveolar $\mathrm{NO}$ concentrations and distal biglycan and perlecan production by fibroblasts from patients with asthma $(r=-0.857, p=0.033$, and $\mathrm{r}=-0.750, \mathrm{p}=0.012$, respectively) (figure $5 \mathrm{~A}, \mathrm{~B}$ ).
Figure 5 Biglycan and perlecan production by distally derived fibroblasts from subjects with asthma was plotted against alveolar nitric oxide (NO) concentration (A and B, respectively) in subjects with asthma (filled circle). Spearman correlation was used to calculate the significance of the degree of association between proteoglycan production and alveolar NO concentration.
A

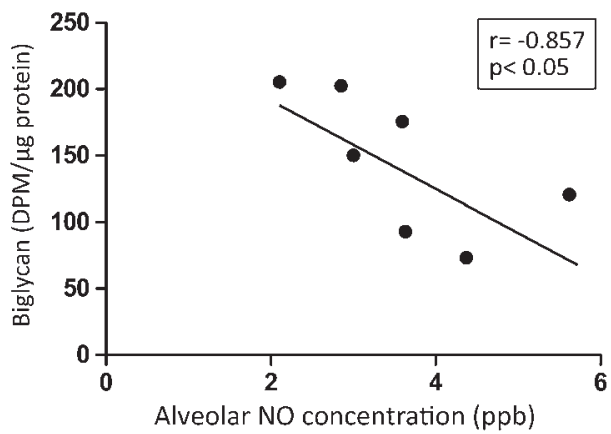

B

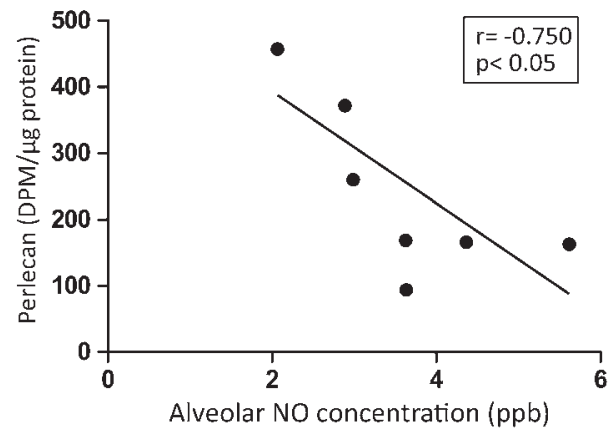




\section{Asthma fibroblasts respond less to TGF $\beta 1$ stimulation than those from controls}

TGF $\beta 1$ significantly increased the production of the proteoglycans versican, biglycan and perlecan in both centrally and distally derived control fibroblasts compared with unstimulated cells (centrally derived $(p=0.016, p=0.016$ and $p=0.039$, and distally derived $(p=0.039, p<0.001$ and $p=0.034)$, respectively) (table 2). In centrally derived asthma fibroblasts, versican production was increased compared with unstimulated cells $(p=0.039)$. In distal airways, TGF $\beta 1$ stimulation significantly increased biglycan production from asthma fibroblasts $(p=0.031)$. For individual values after TGF $\beta_{1}$ stimulation, see the supplementary files.

\section{DISCUSSION}

In this study we have demonstrated that both distally and centrally derived fibroblasts from patients with asthma have an altered cell proliferation and production of matrix molecules, such as versican, compared with controls. We also verified distal airway remodelling in subjects with mild asthma, as described by increased collagen accumulation in peripheral airway tissue. We further showed that distally and centrally derived fibroblasts have different phenotypes, an observation that strengthens the hypothesis that the nature of distal airway remodelling differs from brochial remodelling. Moreover, our results also show that production of the proteoglycans perlecan and biglycan can be linked to alveolar exhaled NO levels.

\section{Remodelling in distal lung}

It is well established that airway remodelling and inflammation take place in the subepithelial compartment in central airways of subject with atopy and asthma. ${ }^{420} 21$ It is also known that inflammation occurs in the distal airways and lung parenchyma. ${ }^{22}$ However, this is, to the best of our knowledge, the first study demonstrating remodelling in the distal airways in subjects with mild asthma, as shown by increased deposition of collagen. In this context, the differences in versican production by distal airway fibroblasts in our in vitro experiments further strengthen the hypothesis that fibroblasts also play a role in distal asthma pathophysiology. The present demonstration of remodelling in the distal lung already in mild asthma may be an important observation that possibly has implications for disease progression since remodelling is a feature in both moderate and severe asthma. Higher levels of versican production were only seen in individuals with asthma, suggesting a shift in diseaserelated fibroblast phenotypes. It has been proposed that due to its high chondroitin sulfate/dermatan sulfate content, versican is destructive for the elasticity in the lung by inhibiting elastinbinding protein (EBP), and thus interferes with assembly of the elastic fibres. ${ }^{23}$ Accumulation of versican in those with mild asthma could consequently affect lung stiffness in both the

Table 2 Influence of transforming growth factor $\beta 1$ (TGF $\beta 1$ ) on individual proteoglycan production from centrally and distally derived fibroblasts and related to basal level production ( $0.4 \%$ serum)

\begin{tabular}{lllll}
\hline & Central controls & Central asthma & Distal controls & Distal asthma \\
\hline Versican & $\mathrm{p}=0.016$ & $\mathrm{p}=0.039$ & $\mathrm{p}=0.039$ & $\mathrm{NS}$ \\
Biglycan & $\mathrm{P}=0.016$ & NS & $\mathrm{p}<0.001$ & $\mathrm{p}=0.031$ \\
Perlecan & $\mathrm{p}=0.039$ & $\mathrm{NS}$ & $\mathrm{p}=0.034$ & NS \\
Decorin & NS & NS & NS & NS
\end{tabular}

Wilcoxon signed rank test was used to calculate the significance of increased production in response to TGF $\beta 1$.

Values of $p<0.05$ were regarded as significant. central and the distal airways, and thus directly influence lung function. ${ }^{5}$ The negative correlation of alveolar $\mathrm{NO}$ concentration and the proteoglycans biglycan and perlecan suggests that $\mathrm{NO}$ may also reflect the degree of remodelling. A lower perlecan production might directly influence basement membrane integrity. Furthermore, the ability of perlecan to bind cytokines and affect epithelial adherence and function may also play an important role in distal remodelling processes. ${ }^{24}$

Fibroblast phenotypes in distal airways - a link to exhaled NO? In this study, there was a correlation between the levels of exhaled $\mathrm{NO}$ and proteoglycan production in distal airways. This finding links remodelling to clinical parameters and suggests that $\mathrm{NO}$ may be used as a marker for fibroblast activity. NO, which is a mediator molecule involved in both proinflammatory and antiinflammatory processes, is known to be upregulated in asthma. ${ }^{10}$ Although the production of $\mathrm{NO}$ in patients with asthma is believed to be due to expression of inducible nitric oxide synthase (iNOS) in epithelial cells, the primary source has not been identified yet. NO has previously been suggested to be involved in lung fibrosis and fibroblast differentiation. ${ }^{25} 26$ It is also known that products in the NO cycle can digest glycosaminoglycans into biologically active fragments. This process could possibly be controlled by fibroblasts due to their expression of iNOS, and thus be responsible for the increased $\mathrm{NO}$ production in patients. ${ }^{27} 28$ Elevated levels of $\mathrm{NO}$ have been shown in patients with more severe asthma, and these patients are known to have more evidence of remodelling in the distal airways. ${ }^{29}$ Moreover, alveolar $\mathrm{NO}$ has also been shown to be elevated in those with mild asthma as compared with controls without asthma. ${ }^{19}$

\section{Role of small proteoglycans in airway remodelling}

In this work basal biglycan production was also altered in centrally derived asthma fibroblasts, further supporting the hypothesis that the composition of connective tissue may be changed in mild asthma. Notably, both biglycan and decorin production from distally derived fibroblasts from atopic individuals with rhinitis was significantly lower than in non-atopic controls, which indicates that these individuals have changes in distal connective tissue composition comparable with those in subjects with asthma. This suggests involvement of the upper and distal airways in airway diseases such as seasonal and chronic rhinitis. However, these observations require confirmation in studies with adequate patient numbers for the analyses to be robust. The important role of proteoglycans in asthma, fibrosis, and chronic obstructive pulmonary disease (COPD) and other diseases is well documented, at both the cellular and the physiological level. ${ }^{2} 230$ Previously published data have shown that the small proteoglycans biglycan and decorin induce morphological and cytoskeletal changes in fibroblasts, suggesting that they have a role in conversion of the resident fibroblast to an inflammatory fibroblast phenotype promoting wound healing processes. ${ }^{10}$ This role of biglycan has also been discussed by others in the context of biglycan functioning as a proinflammatory molecule involved in innate immunity. ${ }^{31}$ Expression of proteoglycans in centrally derived tissue sections from atopic subjects and those with fatal asthma and in animal models has previously been studied using immunohistochemistry, further confirming our results. ${ }^{6} 732$ Fibroblast production of collagen, which is the most abundantly expressed extracellular matrix molecule in the lung, was not measured in the present study. However, some of the proteoglycans interact individually with collagen to modulate collagen organisation 
and network formation. ${ }^{33}$ One of the most potent mediators in stimulating collagen and proteoglycan production is TGF $\beta 1$, and its role in human asthma has been extensively reviewed, but there are contradictory results concerning its direct role. Current evidence suggests that expression of TGF $\beta 1$ is upregulated in asthma. ${ }^{34}$ Overall, since no differences in increased proteoglycan production were observed between groups, our results suggests that the fibroblast changes with regard to proteoglycan production are already present in the cells at basal levels, and might only be enhanced by TGF $\beta 1$.

In this study we also observed a shift from a proliferating cell phenotype, as seen in centrally derived fibroblasts from controls, to a resident matrix-producing cell in subjects with asthma, with increased numbers of cellular extensions. This indicates the presence of different fibroblast phenotypes in controls and patients with asthma. It is an interesting observation that might suggest that fibroblasts in asthma acquire a more activated contractile phenotype, although this needs to be investigated further. ${ }^{35}$ Furthermore, our data are in contrast to data published by Wenzel and colleagues where no difference was observed between those with asthma and controls except for localisation. ${ }^{1}$ This could be due to a different experimental set-up as they used postmortem lung material as the source of control tissue.

\section{SUMMARY}

In conclusion, we have shown for the first time that matrix production by both centrally and distally derived fibroblasts is different for those with mild untreated asthma and for controls. In addition, remodelling in distal lung is also present in subjects with mild asthma as visualised by increased collagen. These differences could also be linked to exhaled NO, which suggests an association between connective tissue composition and lung function in those with mild asthma. In the delicate balance of physiologically normal responses to inflammation and the resolving processes that follow, proteoglycans may have a pivotal role in the initiation and maintenance of pathological remodelling in the airways. Further studies in patients with other subtypes of asthma and comparative studies in other lung diseases will be required to define the precise mechanisms of matrix involvement in order to intervene and counteract this process in the human lung.

Acknowledgements The authors thank Lena Thiman for her laboratory skills and technical assistance.

Funding This work was supported by grants from the Swedish Research Council $(11550,7970)$, the Heart and Lung Foundation, Centrala Försöksdjursnämnden (CFN), the Greta and John Kock Foundation, the Alfred Österlund Foundation, the Crafoord Foundation, Riksföreningen mot Rheumatism, the Schyberg Foundation, the Hedberg Foundation, the Gustaf V 80th Fund, Evy and Gunnar Sandberg Foundation and the Medical Faculty of Lund University.

\section{Competing interests None.}

Patient consent Obtained.

Ethics approval This study was conducted with the approval of the the ethics committee of Lund University (LU412-03).

Provenance and peer review Not commissioned; externally peer reviewed.

\section{REFERENCES}

1. Kotaru C, Schoonover KJ, Trudeau JB, et al. Regional fibroblast heterogeneity in the lung: implications for remodeling. Am J Respir Crit Care Med 2006:173:1208-15.

2. Mauad T, Silva LF, Santos MA, et al. Abnormal alveolar attachments with decreased elastic fiber content in distal lung in fatal asthma. Am J Respir Crit Care Med 2004:170:857-62.

3. Bjermer L. History and future perspectives of treating asthma as a systemic and small airways disease. Respir Med 2001:95:703-19.
4. Jeffery PK. Remodeling in asthma and chronic obstructive lung disease. Am J Respir Crit Care Med 2001;164:S28-38.

5. Westergren-Thorsson G, Chakir J, Lafreniere-Allard MJ, et al. Correlation between airway responsiveness and proteoglycan production by bronchial fibroblasts from normal and asthmatic subjects. Int J Biochem Cell Biol 2002;34:1256-67.

6. Huang $\mathbf{J}$, Olivenstein $\mathrm{R}$, Taha $\mathrm{R}$, et al. Enhanced proteoglycan deposition in the airway wall of atopic asthmatics. Am J Respir Crit Care Med 1999;160:725-9.

7. de Medeiros MM.da Silva LF.dos Santos MA, et al. Airway proteoglycans are differentially altered in fatal asthma. J Pathol 2005:207:102-10.

8. Handley CJ, Samiric T, llic MZ. Structure, metabolism, and tissue roles of chondroitin sulfate proteoglycans. Adv Pharmacol 2006;53:219-32.

9. Sheppard D. Transforming growth factor beta: a central modulator of pulmonary and airway inflammation and fibrosis. Proc Am Thorac Soc 2006;3:413-17.

10. Tufvesson E, Westergren-Thorsson G. Biglycan and decorin induce morphological and cytoskeletal changes involving signalling by the small GTPases RhoA and Rac1 resulting in lung fibroblast migration. J Cell Sci 2003;116:4857-64.

11. Meneghin A, Hogaboam CM. Infectious disease, the innate immune response, and fibrosis. J Clin Invest 2007;117:530-8.

12. Maher TM, Wells AU, Laurent GJ. Idiopathic pulmonary fibrosis: multiple causes and multiple mechanisms? Eur Respir J 2007:30:835-9.

13. Nonaka M, Pawankar R, Fukumoto A, et al. Heterogeneous response of nasal and lung fibroblasts to transforming growth factor-beta 1. Clin Exp Allergy 2008; 38:812-21.

14. Pavord ID, Shaw D. The use of exhaled nitric oxide in the management of asthma $J$ Asthma 2008:45:523-31.

15. Jatakanon A, Lim S, Kharitonov SA, et al. Correlation between exhaled nitric oxide sputum eosinophils, and methacholine responsiveness in patients with mild asthma. Thorax 1998:53:91-5

16. Payne DN, Adcock IM, Wilson NM, et al. Relationship between exhaled nitric oxide and mucosal eosinophilic inflammation in children with difficult asthma, after treatment with oral prednisolone. Am J Respir Crit Care Med 2001;164:1376-81.

17. Simons FE. Allergic rhinobronchitis: the asthma-allergic rhinitis link. J Allergy Clin Immunol 1999;104:534-40.

18. Globa Initiative for Asthma. Global strategy for asthma management and prevention. http://www.ginasthma.com (accessed June 2010).

19. Tufvesson E, Aronsson D, Ankerst J, et al. Peripheral nitric oxide is increased in rhinitic patients with asthma compared to bronchial hyperresponsiveness. Respir Med 2007;101:2321-6

20. Chakir J, Laviolette $\mathrm{M}$, Boulet $\mathrm{M}$, et al. Lower airways remodeling in nonasthmatic subjects with allergic rhinitis. Lab Invest 1996;75:735-44.

21. Pepe C, Foley S, Shannon J, et al. Differences in airway remodeling between subjects with severe and moderate asthma. J Allergy Clin Immunol 2005; 116:544-9

22. Bergeron C, Hauber HP, Gotfried M, et al. Evidence of remodeling in peripheral airways of patients with mild to moderate asthma: effect of hydrofluoroalkaneflunisolide. J Allergy Clin Immunol 2005;116:983-9.

23. Merrilees MJ, Ching PS, Beaumont B, et al. Changes in elastin, elastin binding protein and versican in alveoli in chronic obstructive pulmonary disease. Respir Res 2008;9:41

24. Iozzo RV. Basement membrane preoteolycans: from cellar to ceiling. Nat Rev Mol Cell Biol 2005;6:646-56.

25. Vyas-Read S, Shaul PW, Yuhanna IS, et al. Nitric oxide attenuates epithelial-mesenchymal transition in alveolar epithelial cells. Am J Physiol Lung Cell Mol Physiol 2007;293:L212-21.

26. Romanska HM, Ikonen TS, Bishop AE, et al. Up-regulation of inducible nitric oxide synthase in fibroblasts parallels the onset and progression of fibrosis in an experimental model of post-transplant obliterative airway disease. J Pathol 2000:191:71-7.

27. Mani K, Cheng F, Fransson LA. Heparan sulfate degradation products can associate with oxidized proteins and proteasomes. J Biol Chem 2007;282:21934-44.

28. Romanska HM, Polak JM, Coleman RA, et al. iNOS gene upregulation is associated with the early proliferative response of human lung fibroblasts to cytokine stimulation. J Pathol 2002;197:372-9.

29. Berry M, Hargadon B, Morgan A, et al. Alveolar nitric oxide in adults with asthma: evidence of distal lung inflammation in refractory asthma. Eur Respir $\mathrm{J}$ 2005:25:986-91.

30. Westergren-Thorsson G, Hernnas J, Sarnstrand B, et al. Altered expression of small proteoglycans, collagen, and transforming growth factor-beta 1 in developing bleomycin-induced pulmonary fibrosis in rats. J Clin Invest 1993;92:632-7.

31. Schaefer L, Babelova A, Kiss E, et al. The matrix component biglycan is proinflammatory and signals through Toll-like receptors 4 and 2 in macrophages. $J$ Clin Invest 2005:115:2223-33.

32. Reinhardt AK, Bottoms SE, Laurent GJ, et al. Quantification of collagen and proteoglycan deposition in a murine model of airway remodelling. Respir Res 2005;6:30

33. Ferdous Z, Wei VM, lozzo R, et al. Decorin-transforming growth factor interaction regulates matrix organization and mechanical characteristics of three-dimensional collagen matrices. J Biol Chem 2007 7;282:35887-98.

34. Bosse Y, Rola-Pleszczynski M. Controversy surrounding the increased expression of TGF beta 1 in asthma. Respir Res 2007;8:66.

35. Larsen K, Tufvesson E, Malmstrom J, et al. Presence of activated mobile fibroblasts in bronchoalveolar lavage from patients with mild asthma. Am J Respir Crit Care Med 2004;170:1049-56. 